\title{
Evidence for an eye-movement contribution to normal foveal crowding
}

\author{
Harold E. Bedell, PhD, FAAO* \\ University of Houston, College of Optometry, Houston, TX, USA
}

John Siderov, MScOptom, PhD, FAAO

Monika A. Formankiewicz, $\mathrm{PhD}$

Sarah J. Waugh, MScOptom, PhD, FAAO

Senay Aydin, PhD

Department of Vision \& Hearing Sciences, Anglia Ruskin University, Cambridge, UK

* Author to whom correspondence should be addressed: College of Optometry, 505 J. Davis Armistead Building, University of Houston, Houston, TX USA 77204-2020.

E-mail: $\underline{\text { HBedell@Optometry.uh.edu }}$

Fax: 713-743-2053

Figures: 4

Tables: 2 


\begin{abstract}
Purpose. Along with contour interaction, inaccurate and imprecise eye movements and attention have been suggested to contribute to poorer acuity for "crowded" vs. uncrowded targets. To investigate the role of eye movements in foveal crowding, we compared percent correct letter identification for short and long lines of near-threshold letters with different separations.

Methods. Five normal observers read short (4 - 6 letters) and long (10 - 12 letters) lines of nearthreshold, Sloan letters with edge-to-edge letter separations of 0.5, 1 and 2 letter spaces. Percent correct letter identification for the $2-4$ interior letters in short strings and the 8-10 interior letters in long strings was compared to a no-crowding condition.

Results. Letter identification was significantly worse than the no-crowding condition for long letter strings with a separation of 1 letter space, and for both long and short letter strings with a separation of 0.5 letter spaces. Observers more often reported the incorrect number of letters in long than short letter strings, even for a separation of 2 letter spaces. Similar results were obtained during straight-ahead gaze and while viewing in $30-40$ deg left gaze, where 2 of the 5 observers exhibited an increase in horizonal fixational instability.

Conclusions. We argue that lower percent correct letter identification and more frequent errors in reporting the number of letters in long compared to short letter strings reflect an eyemovement contribution to foveal crowding.
\end{abstract}

\title{
Key words.
}

Crowding, contour interaction, visual acuity, eye movement, fixation. 
1 Contour interaction is an impairment of fine spatial tasks, such as visual acuity, that occurs in

2 the presence of nearby flanking contours. ${ }^{1,2}$ In addition to contour interaction, inaccurate and

3 imprecise eye movements and divided or misplaced attention have been suggested to degrade

4 visual acuity when measured with letter charts in amblyopic eyes. ${ }^{1,3-6}$ For example, Flom et al.

5 reported that amblyopic eyes exhibit shallower psychometric functions and poorer visual acuity

6 using ' $\mathrm{S}$ charts', composed of 8 Landolt $\mathrm{C}$ targets surrounded on all sides by tumbling Es, than

7 for individual Landolt Cs surrounded by flanking bars. ${ }^{1}$ Eyes with normal vision exhibit little or

8 no difference in the psychometric function for these types of acuity targets. ${ }^{1,7}$ In the aggregate,

9 the deleterious influences of contour interaction, eye movements and attention on object

10 discrimination and recognition are referred to as crowding. ${ }^{5}$

11 Additional evidence for an effect of eye movement and/or misplaced attention on the

12 measurement of foveal visual acuity comes from comparisons between standard clinical and

13 repeat letter acuity charts. ${ }^{8}$ Repeat letter charts were developed to eliminate the effect of

14 inaccurate eye positioning on the assessment of visual resolution. Indeed, measured visual acuity

15 typically is better using repeat letter charts compared to standard clinical charts in populations

16 with abnormal eye movement control, such as observers with amblyopia, ${ }^{9,10}$ nystagmus, ${ }^{11,12}$

17 central field loss, ${ }^{13,14}$ and young children. ${ }^{8,15}$ In contrast, adults with normal vision exhibit only a

18 very slight acuity improvement, on the order of 1 to 1.5 letters, on repeat compared to standard

19 letter charts. ${ }^{9,11,14}$ Instead of an eye-movement effect, this small acuity improvement in adults

20 could be attributable to probability summation that results from multiple concurrent views of the

21 same letter.

22 In normal adult observers, the range of eye movements during foveal fixation is larger than

23 the spacing between adjacent threshold-sized letters on an acuity chart. ${ }^{16-18}$ Further, the saccadic 
24 eye movements between closely spaced targets within an array typically are neither accurate nor

25 precise. ${ }^{19,20}$ These observations suggest that, during the measurement of visual acuity, normal

26 adults may sometimes fixate on a letter other than the one they are attempting to read. It seems a

27 legitimate question to ask why these fixation errors don't interfere with normal observers' acuity,

28 when measured with standard clinical letter charts compared to repeat letter charts or isolated

29 letters. One reason may be that most modern clinical acuity charts include 5 or fewer letters of

30 the same angular size per line. ${ }^{21-23}$ Normal adults can quickly, confidently, and accurately judge

31 numerosity for up to approximately 5 items within a visual display; ${ }^{24,25}$ they may therefore be

32 able to identify the location of a letter they are attempting to read even when the fovea is directed

33 momentarily somewhere else. For displays that contain more than 5 items, observers are slower

34 to determine numerosity and more likely to misperceive the number of items. ${ }^{24,25}$ These

35 observations suggest that inaccurate fixation and/or misplaced attention within a line of acuity

36 letters may be more likely to produce miscalls if the number of letters on the line is increased.

37 The purpose of this study was to infer the role of eye movements and/or attention in normal

38 foveal crowding by comparing performance for short and long horizontal strings of letters with

39 the same letter-to-letter separation, which should therefore produce equivalent amounts of

40 contour interaction. We assessed foveal letter identification both in straight ahead gaze and while

41 the observers fixated in left lateral gaze, where fixation was expected to become more variable.

42 Objective eye-movement recordings indicated that this latter expectation was fulfilled only 43 partially.

44

45 Materials and Methods.

46 Observers. 
Five adult observers with normal or corrected-to-normal vision participated after granting

48 voluntary written informed consent. Experimental procedures were reviewed and approved by

49 the Ethics Committee of Anglia Ruskin University. Four of the observers were female (3

50 students and one older adult, age range $21-62$ years old), all of whom remained naïve as to the

51 experimental hypotheses that were tested in the study. The fifth, non-naïve observer was author

52 JS, who was 51 years old. All testing was performed monocularly, using the left eye. One of the

53 observers (SA) was emmetropic. The other four observers had refractive corrections in the tested

54 left eye of $-1.75 \mathrm{sph}(\mathrm{JC}),-6.50 /-1.50 \times 100(\mathrm{JH}),-3.00 /-0.50 \times 150(\mathrm{JS})$, and +1.75 sph (NB),

55 which were provided during the experiments by ophthalmic trial lenses. The non-viewing right

56 eye was occluded with an opaque patch.

57 Psychophysical Methods.

58 Observers viewed dark Sloan letters presented on a 19-inch Clinton Monoray monochrome 59 monitor from an optical distance of $9.5 \mathrm{~m}$, after reflection from two mirrors. The screen 60 resolution was 1024 by 768 pixels and the frame rate was $120 \mathrm{~Hz}$. At this viewing distance, the 61 dimensions of the monitor screen corresponded to 2.33 by $1.75 \mathrm{deg}$. Background luminance was $62110 \mathrm{~cd} / \mathrm{m}^{2}$ and letter contrast, calculated using the Weber formula, was -99\%. A letter size 63 corresponding to $-0.2 \log \mathrm{MAR}$ (3.16 min arc) was used in all of the experimental conditions, as 64 each observer identified approximately $75 \%$ of the letters of this size correctly in preliminary, 65 uncrowded trials (see below). The observers viewed the letters foveally under two conditions of 66 gaze, either straight-ahead or, for observers JC, JH, NB and SA, at 40 deg in left gaze. Subject JS 67 could not comfortably sustain fixation at 40 deg left gaze and was tested at 30 deg left gaze 68 instead. For logistical reasons, data collection began in left gaze only after testing in straight69 ahead gaze was completed. Head position was maintained in each gaze position using a head and 
70 chin rest. For the observers who required refractive correction, trial lenses were mounted on a

71 flexible arm, which allowed them to be placed close to the eye and aligned with the visual axis in

72 both straight-ahead and left gaze.

73 Random sequences of Sloan letters, with the stipulation that adjacent letters on the screen

74 were never the same, were presented for an unlimited viewing duration in 3 types of trials. On

75 each short-string trial, 4, 5 or 6 letters were presented at one time, with a horizontal edge-to-edge

76 letter spacing of $0.5,1$ or 2 letter widths (i.e., $1.58,3.16$ or 6.32 min arc). On each long-string

77 trial, a sequence of 10, 11 or 12 letters was presented, also with a horizontal edge-to-edge letter

78 spacing of $0.5,1$ or 2 letter widths. The minimum separation between the edge of the monitor

79 and the edges of the first and last letter in the longest letter strings was 3 letter spaces. For each

80 direction of gaze, observers completed 108 short-string trials (36 each with 4, 5 and 6 letters) and

8136 long-string trials (12 each with 10, 11 and 12 letters). The numbers of short- and long-string

82 trials were chosen so that performance in the two conditions was based on the same total

83 numbers of letters. Short- and long-string trials were presented in blocks of 36 and 12,

84 respectively. Within a block of short- or long-string trials the letter-to-letter separation remained

85 constant at $0.5,1$ or 2 letter widths and the strings with different numbers of letters were

86 presented in a random order. Observers were instructed to read all of the letters in each string in

87 sequence from left to right. Although the observers were informed that different numbers of

88 letters would be presented on different trials, they were not told about the length of each letter

89 string either prior to or after a trial. A single trial lasted between approximately 5 and $15 \mathrm{~s}$ and

90 each block of 12 or 36 trials had a total duration of between 5 - 10 minutes. The experimenter

91 used the computer keyboard to record the observers' letter responses in order and subsequently

92 compared the responses to the presented sequences of letters. Because crowding is reduced for 
93 letters at the left and right ends of a line, ${ }^{26,27}$ performance was calculated by scoring only the

94 interior 2, 3 or 4 letters for the trials with 4, 5, and 6 letters, respectively, and only the interior 8 ,

959 or 10 letters for the trials with 10, 11, and 12 letters. Letters had to be read in the correct order

96 to be scored as correct.

97 For comparison with the short- and long-string trials, each observer first completed six

98 blocks (one for each combination of letter separation and gaze direction) of 34 uncrowded trials.

99 On each uncrowded trial, three letters were presented with an edge-to-edge spacing of 5 letter

100 widths (i.e., $15.8 \mathrm{~min}$ arc), a distance well beyond the extent of foveal crowding. ${ }^{1,6,28}$ Because

101 crowding was assumed to be absent in this condition, all 3 letters presented on each trial were

102 scored. As above, the letters had to be read in the correct order to be scored as correct.

103 Eye Movement Recording.

104 To evaluate the influence of gaze direction on fixational eye movements, the horizontal and

105 vertical positions of each observer's left eye were recorded at a sampling rate of $250 \mathrm{~Hz}$ using an

106 EyeLink 1000 Eyetracker with remote camera. Eye movement recordings were obtained in a

107 separate session from the psychophysical measurements. During each trial, the observer fixated

108 for $20 \mathrm{~s}$ at the center of a 3 to 5 letter string, displayed at a distance of $1 \mathrm{~m}$ on a 19-inch Sony

109 Trinitron monitor. Four recordings were made while the observer viewed in straight-ahead gaze

110 and another four were made during foveal fixation at $40 \mathrm{deg}$ (observers JC, JH, NB and SA) or

$11130 \mathrm{deg}$ (observer JS) left gaze. For each direction of gaze, head position was maintained using a

112 head and chin rest.

113 Subsequently, two 5-s intervals without blinks were selected from each fixation file for

114 further analysis: (1) calculation of the standard deviations (SDs) of the horizontal and vertical 115 eye positions during each interval and (2) counts of the number of fixational saccades. The eye 
116 movement results for each observer were summarized by taking the mean horizontal and vertical

117 SDs and the mean number of fixation saccades during the 8 separate fixation intervals (i.e., 4

118 fixation trials $\mathrm{x} 2$ intervals per trial).

119 Data Analysis.

120 To perform statistical analyses, the observers' percent correct letter identification for each

121 experimental condition was converted to a $\mathrm{z}$ score, as between-observer differences in $\mathrm{z}$ scores

122 for the different experimental conditions are more similar than the differences in percent correct.

123 A three-way repeated-measures ANOVA assessed the effects of string length (single letters,

124 short strings and long strings), letter separation (0.5, 1 and 2 letter widths), and gaze direction

125 (straight-ahead and left gaze). A separate two-way repeated-measures ANOVA evaluated 126 whether string length and direction of gaze affected the percentage of trials on which the 127 observers reported the correct number of letters. A Huynh-Feldt correction was applied to 128 statistical tests when a departure from sphericity occurred. Although the statistical analyses were 129 performed using $\mathrm{z}$ scores, for ease of presentation the results were transformed back to percent 130 correct letter identification when plotting Figures $1-3$, below.

\section{Results.}

132 Letter Identification.

133 The average $\mathrm{z}$ scores for letter identification in the uncrowded condition were 0.64 in 134 straight-ahead gaze and 0.43 in left gaze, corresponding to $73.8 \%$ and $66.5 \%$ correct, 135 respectively. These percentages did not differ significantly according to the letter position in the 136 three-letter array (range: $72.0 \%-74.3 \%$ in straight-ahead gaze; $63.4 \%-66.6 \%$ in left gaze), 137 confirming the absence of contour interaction in this condition. When the edge-to-edge 138 separation between adjacent letters was 2 letter spaces, performance in the short- and long-string 
139 conditions did not differ from that in the uncrowded condition (Figure 1 top panels). However,

140 letter identification was poorer when the separation was less than 2 letter spaces, as indicated by

141 a significant effect of separation $\left(\mathrm{F}_{\mathrm{df}=2,8}=16.95, \mathrm{p}=0.0013\right)$. The ANOVA also revealed a

142 significant 2 -way interaction between letter separation and string length $\left(\mathrm{F}_{\mathrm{df}=4,16}=10.53, \mathrm{p}=\right.$

143 0.011). Compared to the uncrowded condition, $\mathrm{z}$ scores were lower for long letter strings when

144 the separation was 1 letter space $\left(\mathrm{F}_{\mathrm{df}=1,16}=13.19, \mathrm{p}=0.019\right)$, and for both the long and short

145 letter strings when the separation was 0.5 letter spaces (for long letter strings, $F_{d f=1,16}=61.81, p=$

146 0.0006; for short letter strings, $\left.F_{d f=1,16}=29.08, p=0.0039\right)$. These effects are visible in the data

147 collected both in straight-ahead and left gaze in the middle and lower panels of Figure 1. Figure

1482 replots the percent correct letter identification in straight ahead and left gaze, averaged across

149 subjects, as a function of the letter separation and shows clearly that the magnitude of crowding

150 is greater for the long compared to short letter strings.

151 Neither the direction of gaze nor the interactions between gaze direction and letter separation

152 or string length exhibited a significant effect on letter identification. However, the ANOVA

153 revealed a significant 3-way interaction between direction of gaze, letter separation, and string-

154 length $\left(\mathrm{F}_{\mathrm{df}=4,16}=3.03, \mathrm{p}=0.049\right)$. Means comparisons indicated that this interaction resulted

155 from significantly better performance in straight-ahead compared to left gaze for long strings

156 when the letter separation was 2 letter widths $\left(\mathrm{F}_{\mathrm{df}=1,16}=13.56, \mathrm{p}=0.0020\right)$.

157 In a second analysis, we compared the percentage of trials (again, converted to $\mathrm{z}$ scores) on

158 which the observers reported the correct numbers of letters in the short- and long-string

159 conditions. As anticipated, a three-way repeated-measures ANOVA found that observers

160 reported the correct number of letters less frequently on long-string than short-string trials $\left(\mathrm{F}_{\mathrm{df}=1,4}\right.$

$161=145.96, \mathrm{p}=0.0003)$. Observers also reported the correct number of letters less often as the 
162 separation between adjacent letters decreased from 2 to 0.5 letter widths $\left(\mathrm{F}_{\mathrm{df}=1,4}=26.41, \mathrm{p}=\right.$

163 0.0014). These trends can be seen in the results for both straight-ahead and left gaze, which are

164 plotted in Figure 3. Of particular interest is the observation that observers reported the correct

165 number of letters on a significantly lower proportion of long-compared to short-string trials even

166 with a separation of two letter widths $\left(\mathrm{F}_{\mathrm{df}=1,8}=12.59, \mathrm{p}=0.0075\right)$. Although the observers

167 generally reported the correct number of letters less frequently in left lateral compared to 168 straight-ahead gaze (Figure 3 ), this difference did not achieve statistical significance $\left(\mathrm{F}_{\mathrm{df}=1,4}=\right.$ $1696.05, \mathrm{p}=0.070)$. More detailed information is presented in Table 1, which summarizes the 170 percentage of trials on which the observers reported fewer or more than the correct number of 171 letters for each of the experimental conditions. Overall, when the observers did not report the 172 correct number of letters, they erred more often $(\sim 60: 40)$ by reporting too few rather than too 173 many letters, both for the short and long letter strings.

174 When the observers reported too few letters, the majority of the time they reported one letter 175 too few (73\% of errors for long strings; $97 \%$ of errors for short strings). Similarly, when the 176 observers reported too many letters, on the majority of trials they reported one extra letter $(88 \%$ 177 of trials for long strings; $98 \%$ of trials for short strings). On the remainder of the trials when an 178 incorrect number letters was reported, the observers either omitted or added 2 (13\% of trials) or 3 179 letters (1.6\% of trials), primarily on long-string trials.

180 Examination of the responses on trials when the observers reported the incorrect number of 181 letters indicated that errors of omission or insertion occurred almost always at interior positions 182 in the string. The modal locations of these errors were letter places 6 (omissions) and 8 183 (insertions) in the long strings (accumulated across strings of 10, 11 and 12 letters), and place 4 184 (omissions and insertions) in the short letter strings (accumulated across strings of 4, 5, and 6 
185 letters). When the observers reported one or more extra letters within a string, they typically 186 reported a pair of confusion letters for one letter within the string, such as both ' $N$ ' and ' $R$ ' for 187 the occurrence of an ' $\mathrm{R}$ ' or both ' $\mathrm{D}$ ' and ' $\mathrm{C}$ ' for the occurrence of an 'O.'

188 Observers would be expected to make more errors in reporting the correct number of letters 189 for long compared to short letter strings, simply because the long strings include more letters to 190 report. However, the following analysis reveals that merely increasing the average number of 191 letters in the string does not account for our results. Assume the probability of incorrectly 192 reporting each letter, either by omitting the letter or reporting an additional letter, is the same 193 regardless of the string length. Further, assume that this probability, which we will designate as $194 \mathrm{p}_{\mathrm{e}}$, depends on the separation between the adjacent letters in the string. For each letter separation, 195 we can estimate the value of $p_{e}$ from the percentage of reporting errors made when reading short 196 letter strings, $\mathrm{p}_{5}$. Specifically, if each short letter string is assumed to comprise 5 letters, then

$$
\left(1-\mathrm{p}_{\mathrm{e}}\right)^{5}=1-\mathrm{p}_{5}
$$

198 and the predicted percentage of errors when reading strings of 11 letters, $\mathrm{p}_{11}$, is

$$
\mathrm{p}_{11}=1-\left(1-\mathrm{p}_{\mathrm{e}}\right)^{11}
$$

200 For example, when the letter separation is 2 letters in straight-ahead gaze, summing the 201 percentages of reporting too few and too many letters in the short-string condition yields $\mathrm{p}_{5}=$ $2020.023($ Table 1$)$ and $\left(1-\mathrm{p}_{5}\right)=0.977$. Based on this result, the predicted probability of reporting 203 an incorrect number of letters in the analogous long-string condition, $\mathrm{p}_{11}$, is 0.05 . The observed 204 probability of reporting the incorrect number of letters in this long-string condition was 0.15 , 205 which is significantly higher $(\mathrm{z}=3.55, \mathrm{p}=0.0004)$ than the predicted value. The observed 206 probabilities of reporting the incorrect numbers of letters in the long-string condition also is 
207 significantly greater than the predicted probabilities $(\mathrm{p}<0.0001)$ for the other 5 conditions of

208 letter separation and gaze direction.

209 Fixational Eye Movements.

210 During fixation in straight-ahead gaze, the average standard deviations (SD) of both the

211 horizontal and the vertical eye position measurements were approximately $0.16 \mathrm{deg}$ (i.e., $9.5 \mathrm{~min}$

212 arc; Table 2). Although four of the five observers (all except JH) exhibited a small endpoint

213 nystagmus when fixating in left gaze (Figure 4), only two of them exhibited a noticeable increase

214 in the SD of horizontal eye position: for observer JS from 0.13 to $0.19( \pm 0.02 \mathrm{SE})$ deg and for

215 observer NB from 0.19 to $0.27( \pm 0.02 \mathrm{SE})$ deg. The SDs of vertical eye position were either

216 unchanged in left compared to straight-ahead gaze or decreased (from 0.24 to 0.11 deg for

217 observer JC). However, consistent with the observed endpoint nystagmus in left gaze, the

218 average number of saccades during fixation in left gaze was significantly greater than the number 219 of saccades during fixation in straight-ahead gaze (mean difference $=2.58$ saccades $/ 5 \mathrm{~s}, \mathrm{t}_{\mathrm{df}=4}=$ $2203.10, \mathrm{p}=0.036)$.

221 We compared the change in correct letter identification between straight-ahead and left gaze

222 in observers JS and NB, who showed an increase in the SD of horizontal eye position, to the

223 change in performance of the other three observers. We focused on the change in $\mathrm{z}$ scores for

224 long letter strings separated by two letter widths because, as reported above, this condition

225 produced a significant gaze-dependent effect on crowding. The average change in z score from

226 straight ahead to left gaze was $0.75 \pm 0.08$ for JS and NB, compared to $0.10 \pm 0.19$ for the other

227 three observers.

228 Discussion. 
In normal observers, contour interaction does not occur in the fovea for targets separated by

230 more than 3-6 min arc, which corresponds approximately to the width of a high-contrast

231 threshold acuity letter. ${ }^{1,2,7,29-31}$ On this basis, we conclude that only the immediately adjacent

232 letters within each string contributed to contour interaction and that the magnitude of contour

233 interaction was identical for the interior letters in the short- and long-string stimuli in our

234 experiment. The greater magnitude of crowding that we observed for strings of 10, 11 and 12

235 letters than for strings of 4, 5 and 6 letters for a separation of 0.5 and 1 letter space (Figure 2)

236 therefore can not be attributed to an increase in contour interaction.

237 Inaccurate and imprecise eye movements are a potential contributor to foveal crowding. ${ }^{1,5,8,32}$

238 The imprecision of normal fixation is large enough to encompass more than a single letter, even

239 in the letter strings with a separation of two letter spaces between the adjacent letters ${ }^{16-18}$ (see

240 also Figure 4). Moreover, it is highly unlikely that our observers accurately imaged the

241 successive letters in the strings sequentially on the fovea. Kowler and Steinman ${ }^{20}$ measured the

242 saccades made by two experienced observers when counting 10 to 19 identical vertical lines

243 separated horizontally by gaps of $7.4-14.2 \mathrm{~min}$ arc. For lines separated by 7.4 to $8.2 \mathrm{~min}$ arc,

244 one observer made saccades that ranged in amplitude from approximately 7 to 45 min arc; the

245 saccades of the other observer ranged from less than 5 to approximately 20 min arc. Both

246 observers frequently lost their place while counting the vertical lines and, for the range of line

247 separations examined, reported the correct number of lines on half or fewer of the trials. Our

248 finding that observers often reported the incorrect number of letters in the long-string condition

249 (Fig. 3) is consistent with this observation. As suggested above in the Introduction, observers

250 may be less likely to lose their place in short letter strings because they can judge the number of 
251 letters, and presumably their relative positions in the string, essentially instantaneously and 252 effortlessly. ${ }^{24,25}$

253 Liu and Arditi observed that normal observers frequently reported only 4 of the letters in 254 strings of 5 upper case letters when the edge-to-edge letter-to-letter spacing was made very 255 small. $^{33}$ The frequency of these under-reporting errors dropped rapidly from approximately $80 \%$ 256 to $20 \%$ as the separation between adjacent letters increased from 0.04 to 0.24 of the threshold 257 letter size. Only rarely did the observers make the opposite error of reporting 5 letters in strings 258 that were 4 letters in length. Based on the strong dependence of under-reporting errors on the 259 letter separation, the authors attributed these errors to overlap between the retinal images of 260 adjacent letters. The observers in our study often erred in reporting the correct number of letters 261 for edge-to-edge separations of 0.5 and 1 letter width, well beyond the range of separations used 262 by Liu and Arditi. ${ }^{33}$ In addition, both under- and over-reporting of the number of letters were 263 common among our observers (Table 1). For these reasons, we conclude that our results can not 264 be attributed to overlap between the images of adjacent letters.

265 It is possible that the observers used the angular extent subtended by each string as a cue to 266 the number of letters, as the ratios of the extent for the different short and long strings presented 267 within a single block of trials generally exceed the Weber fraction for length discrimination (2 $2685 \%$, e.g., Helmholtz, 1925; Ono, $1967^{34,35}$ ). For the three letter separations tested, the ratios of 269 extent for the 4, 5, and 6 letter strings are greater than those between 10, 11, and 12 letter strings, 270 which potentially could account for the higher percentage of errors in reporting the number of 271 letters on long- compared to short-string trials. However, the ratios of angular extent for the 272 short and long letter strings do not change appreciably with the letter separation; i.e., the angular 273 extent of 6 and 4 letter strings are in the ratio of 1.60 for a letter separation of two spaces and 
2741.55 for a letter separation of 0.5 spaces. Similarly, the angular extent of 12 and 10 letter strings

275 are in the ratio of 1.21 for letter separations of both two and 0.5 spaces. Nevertheless, the

276 number-of-response errors increase as the letter separation decreases from two to 0.5 spaces,

277 both for the short and long letter strings (Figure 3). Further, in addition to the angular extent of

278 the letter strings, information about the string length on each trial was available also from the

279 angular separation between the first and last letter of the string and the edges of the monitor

280 screen. The ratios of these separations for the different string lengths are uniformly higher for

281 long than for short letter strings, and are greatest for long strings when the letter separation is

282 large. When the separation between the adjacent letters in the long strings was two spaces, the

283 ratios of the distances to the monitor edge for strings of 10,11 , and 12 letters are essentially

284 identical to the ratios of the angular extents for strings of 4,5 , and 6 letters separated by one

285 space. Nevertheless, observers made approximately 4.5 times more number-of-response errors

286 on long-string trials with a separation of two letter spaces than on short-string trials with a

287 separation of one letter space. The observations in this paragraph suggest that the observers did

288 not attempt to deduce the number of letters presented on each trial and that, for a given

289 separation between letters, the more frequent errors for long compared to short strings in the

290 number of reported letters is attributable primarily to the number of letters the in the string.

291 We expected that observers would make more errors when they viewed the letter strings at

29230 or 40 deg in left gaze compared to straight ahead because of an increase in the variability of

293 fixation in association with the development of endpoint nystagmus. ${ }^{36-38}$ Although we observed a

294 trend for a greater number of miscalls and more frequent errors in reporting the number of letters

295 in a string, these trends did not reach statistical significance. The likely reason for the observers'

296 overall similar psychophysical performance in left and straight-ahead gaze is that the variability 
297 of horizontal fixation did not change significantly in left gaze for three of the five observers. The

298 two observers whose horizontal fixation was less stable in left gaze also identified widely spaced

299 letters in the long-string condition less accurately in left compared to straight-ahead gaze. It is

300 possible that fixation would have been less steady and psychophysical performance would have

301 been worse if the observers had been required to view the letters with gaze diverted further to

302 one side. $^{38}$

303 A displacement of attention from the acuity target towards the flanking stimuli also has been

304 suggested to contribute to crowding. ${ }^{1,5}$ Leat et al. sought to assess the influence of attention on

305 crowding by varying the similarity of the flanking targets to one another and to the acuity target

306 which, in their study, was a Landolt $\mathrm{C}$ or tumbling E. ${ }^{6}$ The results indicated that the manipulation

307 of flanker characteristics generates no consistent change in the magnitude of foveal crowding.

308 However, it may be difficult to tease apart the potentially separate influences of eye movements

309 and attention on foveal crowding, as there is evidence to suggest that the locus of attention

310 remains closely tied to the direction of gaze during large eye movements. ${ }^{39-41}$ A similar intimate

311 relationship has been suggested to exist also between the direction of gaze and attention during

312 small shifts in the position of fixation by some, ${ }^{42-44}$ but not all authors. ${ }^{45}$

313 We conclude that both the greater magnitude of foveal crowding and more frequent errors in

314 reporting the correct number of letters in long compared to short letter strings are attributable to

315 the imprecision and inaccuracy of small normal eye movements. As surmised previously by

316 others, the eye-movement contribution to crowding may be substantially greater in individuals

317 whose eye-movement control is poor, such as in patients with nystagmus, ${ }^{11,12}$ amblyopia, ${ }^{1,5,9,10}$

318 and young children. , $^{8,15,32}$

319 Acknowledgments. 
320 This research was supported by a grant from the Evelyn Trust and a Leverhulme Visiting

321 Professorship to Anglia Ruskin University. Portions of the data were presented at the annual

322 meeting of the American Academy of Optometry in Seattle, WA in October, 2013.

323 


\section{References.}

325 1. Flom MC, Weymouth FW, Kahneman D. Visual resolution and contour interaction. Journal of the 326 Optical Society of America, 1963; 53:1026-32.

327 2. Takahashi ES. Effects of flanking contours on visual resolution at foveal and near-foveal loci. PhD

328 Thesis: School of Optometry, Berkeley: University of California;1968.

329 3. Adler FH. Physiology of the Eye: Clincal Application, $3^{\text {rd }}$ Edition. St. Louis, Mosby, 1959:697-8.

330 4. Flom MC. New concepts in visual acuity. Optometric Weekly 1966; 57(28): 63-8.

331 5. Flom MC. Contour interaction and the crowding effect. Problems in Optometry 1991; 3: 237-57.

332 6. Leat SJ, Li W, Epp K. Crowding in central and eccentric vision: the effects of contour interaction and 333 attention. Investigative Ophthalmology and Visual Science 1999; 40: 504-12.

334 7. Danilova MV, Bondarko VM Foveal contour interactions and crowding effects at the resolution limit of the visual system. Journal of Vision 2006; 7(2); 25:1-18.

8. Kothe AC, Regan D. Crowding depends on contrast. Optometry and Vision Science 1990; 67: 283-6.

9. Regan D, Giaschi DE, Kraft SP, Kothe AC. Method for identifying amblyopes whose reduced line acuity is caused by defective selection and/or control of gaze. Ophthalmic \& Physiological Optics, 1992; 12: 425-32.

10. Simmers AJ, Gray LS, McGraw PV, Winn B. Functional vision loss in amblyopia and the effect of occlusion therapy. Investigative Ophthalmology \& Visual Science 1999; 40: 2859-71.

342 11. Simmers AJ, Gray LS, Winn B. The effect of abnormal fixational eye movements upon visual acuity in congenital nystagmus. Current Eye Research 1999; 18: 194-202.

344 12. Park H, Robins D, Guyton DL. A new way to measure potential visual acuity in nystagmus patients:

345 full-field E test. Investigative Ophthalmology \& Visual Science 2010; 51, E-Abstract \#1996.

346 13. Harris MJ, Robins D, Dieter Jr JM, Fine SL, Guyton DL. Eccentric visual acuity in patients with 347 macular disease. Ophthalmology 1985; 92: 1550-3.

348 14. Pardhan S. Crowding in visually inpaired patients: contour interaction and/or gaze selection defects? $349 \quad$ Neuro-ophthalmology 1997; 18: 59-65.

350 15. Simmers AJ, Gray LS, Winn B. Visual function thresholds in children. Current Eye Research 2000; $351 \quad 21: 616-26$.

352 16. Cherici C, Kuang X, Poletti M, Rucci M. Precision of sustained fixation in trained and untrained 353 observers. Journal of Vision 2012; 12(6);31: 1-16. 
354 17. Ferman L, Collewijn H, Jansen TC, Van den Berg AV. Human gaze stability in the horizontal,

355 vertical and torsional direction during voluntary head movements, evaluated with a three-dimensional 356 scleral induction coil technique. Vision Resesarch 1987; 27: 811-28.

357 18. Skavenski AA, Hansen RH, Steinman RM, Winterson B. Quality of retinal image stabilization during 358 small natural and artificial body rotations in man. Vision Research 1979; 19: 675-83.

359 19. He P, Kowler E. The role of saccades in the perception of texture patterns. Vision Research 1992; 32: $360 \quad 2151-63$.

361 20. Kowler E, Steinman RM The role of small saccades in counting. Vision Research 1977; 17: 141-6.

362 21. Bailey IL, Lovie JE. New design principals for visual acuity letter charts. American Journal of 363 Optometry \& Physiological Optics 1976; 53: 740-5.

364 22. Ferris $3^{\text {rd }}$ FL, Kassoff A, Bresnick GH, Bailey I. New acuity charts for clinical research. American 365 Journal of Ophthalmology, 1982; 94: 91-6.

366 23. Hartmann EE, Dobson V, Hainline L, Marsh-Tootle W, Quinn GE, Ruttum MS, Schmidt PP, Simons 367 K. Preschool vision screening: summary of a task force report. Pediatrics, 2000; 106: 1105-12.

368 24. Kaufman EL, Lord MW, Reese TW, Volkmann J. The discrimination of visual number. American 369 Journal of Psychology 1949; 62: 498-525.

370 25. Li R, MacKeben M, Chat SW, Kumar M, Ngo C, Levi DM. Aging and visual counting. PLoS One $371 \quad 2010 ; 5 ; \mathrm{e} 13434: 1-8$.

372 26. Mathew JA, Shah SS, Simon JW. Varying difficulty of Snellen letters and common errors in 373 amblyopic and fellow eyes. Archives of Ophthalmolgy 2011; 129: 184-7.

374 27. Stuart JA, Burian HM. A study of separation difficulty. Its relationship to visual acuity in normal and 375 amblyopic eyes. American Journal of Ophthalmology 1962; 53: 471-7.

376 28. Hess RF, Dakin SC, Kapoor N, Tewfik M. Contour interaction in fovea and periphery. Journal of the 377 Optical Society of America A 2000; 17: 1516-24.

378 29. Bedell HE, Siderov J, Waugh SJ, Zemanova R, Pluhacek F, Musilova L. Contour interaction for 379 foveal acuity targets at different luminances. Vision Research 2013; 89: 90-5.

380 30. Siderov J, Waugh SJ, Bedell HE. Foveal contour interaction for low contrast acuity targets. Vision $381 \quad$ Research 2013; 77: 10-3.

382 31. Lev M, Yehezkel O, Polat U. Uncovering foveal crowding? Scientific Reports 2014; 4: 1-6.

383 32. Norgett Y, Siderov J. Crowding in children's visual acuity tests - effect of test design and age.

384 Optometry \& Vision Science 2011; 88: 920-27. 
33. Liu L, Arditi A. Apparent string shortening comcomitant with letter crowding. Vision Research 2000; 40: $1059-67$.

34. von Helmholtz, H. Treatise on Physiological Optics. Volume III. The Perceptions of Vision. Southall, JPC, trans. New York: Optical Society of America; 1925: 169-170, 232-233.

35. Ono, H. Difference thresholds for stimulus length under simultaneous and nonsimultaneous viewing conditions. Perception \& Psychophysics 1967; 2: 201-207.

36. Abel LA, Parker L, Daroff RB, Dell'Osso LF. End-point nystagmus. Investigative Ophthalmology \& Visual Science 1978; 17, 539-44.

37. Eizenman M, Cheng P, Sharpe JA, Frecker RC. End-point nystagmus and ocular drift: an experimental and theoretical study. Vision Research 1990; 30: 863-77.

38. Shallo-Hoffmann J, Schwarze H, Simonsz HJ, Mühlendyck H. A rexamination of end-point and rebound nysatgmus. Investigative Ophthalmology \& Visual Science 1990; 31: 388-92.

39. Rizzolatti G, Riggio L, Dascola I, Umiltá, C. Reorienting attention across the horizontal and vertical meridians: evidence in favor of a premotor theory of attention. Neuropsychologia 1987; 25: 31-40.

40. Kowler E, Anderson E, Dosher B, Blaser E. The role of attention in the programming of saccades. Vision Research 1995; 35: 1897-1916.

41. Deubel H, Schneider WX. Saccade target selection and object recognition: evidence for a common attentional mechanism. Vision Research 1996; 36: 1827-37.

42. Engbert R, Kliegl R. Microsaccades uncover the orientation of covert attention. Vision Research 2003; 43: 1035-45.

43. Hafed ZM, Clark JJ. Microsaccades as an overt measure of covert attention. Vision Research 2002; 42: $2533-45$.

44. Hafed ZM, Lovejoy LP, Krauzlis RJ. Modulation of microsaccades in monkey during a covert visual attention task. Journal of Neuroscience 2011; 31: 15219-30.

45. Horowitz TS, Fine EM, Fencsik DE, Yurgenson S, Wolfe JM. Fixational eye movements are not an 
412 Table 1. Percentages of trials on which the five observers reported too few vs. too many letters

413 for different combinations of string length, letter spacing, and gaze direction.

414

Letter Type of Straight-ahead Gaze Left Gaze

Separation Error Short strings Long strings Short strings Long strings

\begin{tabular}{|c|c|c|c|c|c|}
\hline \multirow[t]{2}{*}{0.5} & too few & $18.9 \%$ & $40.7 \%$ & $10.6 \%$ & $45.0 \%$ \\
\hline & too many & $4.4 \%$ & $20.0 \%$ & $7.2 \%$ & $30.0 \%$ \\
\hline \multirow[t]{2}{*}{1} & too few & $2.2 \%$ & $35.0 \%$ & $1.7 \%$ & $36.7 \%$ \\
\hline & too many & $1.1 \%$ & $20.0 \%$ & $4.4 \%$ & $16.7 \%$ \\
\hline \multirow[t]{2}{*}{2} & too few & $0.6 \%$ & $1.7 \%$ & $0.6 \%$ & $15.0 \%$ \\
\hline & too many & $1.7 \%$ & $13.3 \%$ & $2.2 \%$ & $13.3 \%$ \\
\hline
\end{tabular}

415

416

417

418

419

420

Table 2. Mean standard deviations (SDs) of horizontal and vertical eye position during fixation for five observers in straight-ahead and left gaze.

\begin{tabular}{|c|c|c|c|c|}
\hline \multirow[b]{2}{*}{ Observer } & \multicolumn{2}{|c|}{ Straight-ahead Gaze } & \multicolumn{2}{|l|}{ Left Gaze } \\
\hline & Horizontal SD & Vertical SD & Horizontal SD & Vertical SD \\
\hline $\mathrm{JC}$ & $0.16 \pm 0.05$ & $0.24 \pm 0.09$ & $0.14 \pm 0.04$ & $0.11 \pm 0.02$ \\
\hline $\mathrm{JH}$ & $0.17 \pm 0.05$ & $0.17 \pm 0.03$ & $0.17 \pm 0.04$ & $0.15 \pm 0.01$ \\
\hline JS & $0.13 \pm 0.04$ & $0.11 \pm 0.04$ & $0.19 \pm 0.03$ & $0.14 \pm 0.06$ \\
\hline NB & $0.19 \pm 0.03$ & $0.10 \pm 0.04$ & $0.27 \pm 0.04$ & $0.13 \pm 0.04$ \\
\hline SA & $0.17 \pm 0.05$ & $0.18 \pm 0.11$ & $0.13 \pm 0.03$ & $0.17 \pm 0.03$ \\
\hline
\end{tabular}




\section{Figure Legends.}

424

Figure 1. Percent correct letter identification is plotted for 5 observers for 3 presentation conditions of foveal viewing: uncrowded ('single') letters, short-letter strings, and long-letter strings. The top, middle, and lower pairs of panels show data for edge-to-edge letter separations of 2, 1 and 0.5 letter spaces. The right and left columns present results for letter strings viewed in straight ahead and left gaze, respectively. Within each column, the same values are plotted for each observer in the three panels for the uncrowded condition. The black lines in each panel connect the mean values of percent correct for the 3 different presentation conditions.

Figure 2. Mean percent correct letter identification is plotted as a function of the edge-to-edge letter spacing for short- and long-letter strings. The values plotted for a separation of 5 letter widths are from the uncrowded condition. The top and bottom panels present results for letters viewed in straight-ahead and left gaze.

Figure 3. The percentages of trials on which the observers reported the correct numbers of letters in the short and long letter strings are plotted as a function of the edge-to-edge letter separation within the strings. Results are shown separately for straight-ahead (unfilled symbols) and left gaze (filled symbols).

Figure 4. Five-second recordings of horizontal (black, higher traces) and vertical (gray, lower traces) eye position during fixation in straight-ahead (top) and left gaze (bottom) are presented for observer JC. The placement of each trace with respect to the vertical axis is arbitrary. Upward deflections represent rightward and upward eye movement. A leftward endpoint nystagmus with an amplitude of approximately one third of a degree is visible in the lower panel. 
2 Letter Spaces

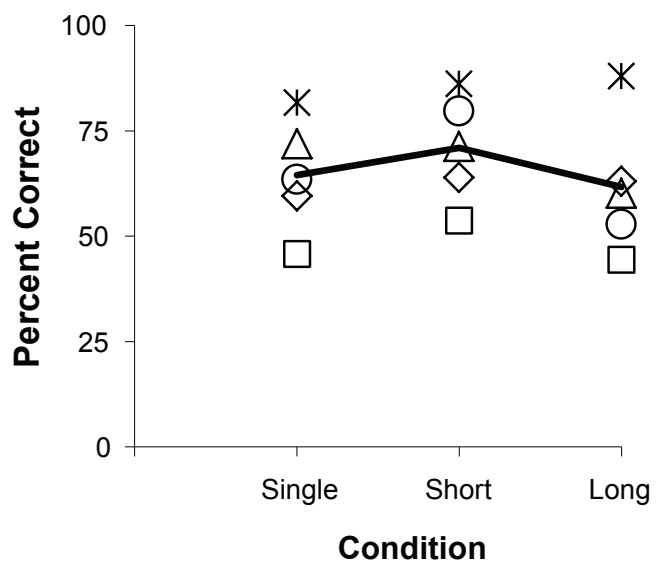

1 Letter Space

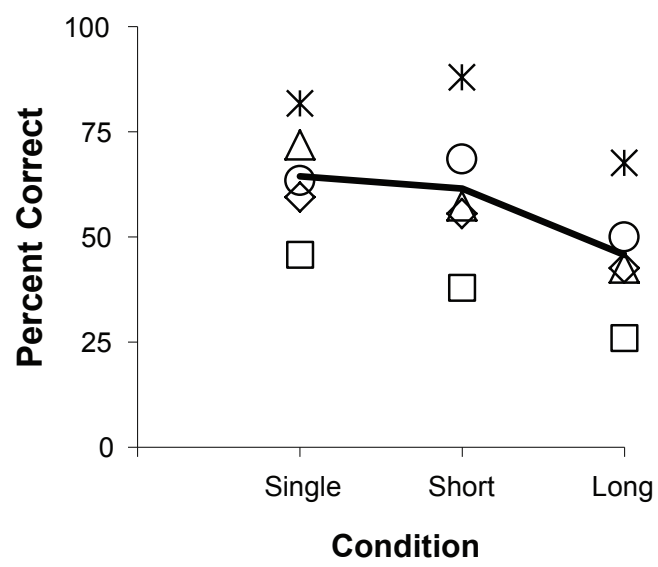

0.5 Letter Spaces

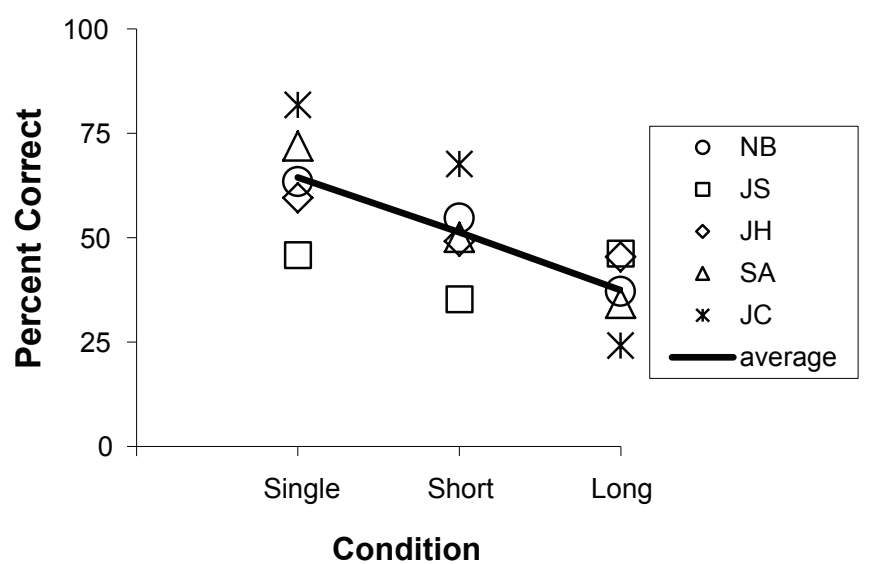

2 Letter Spaces

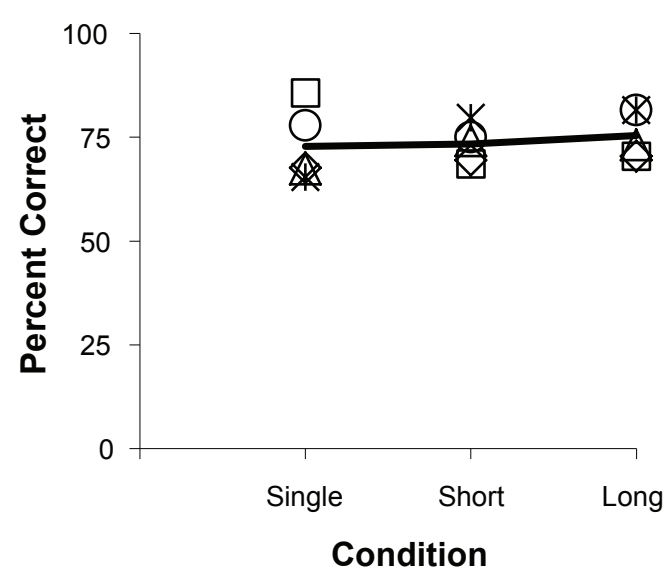

1 Letter Space

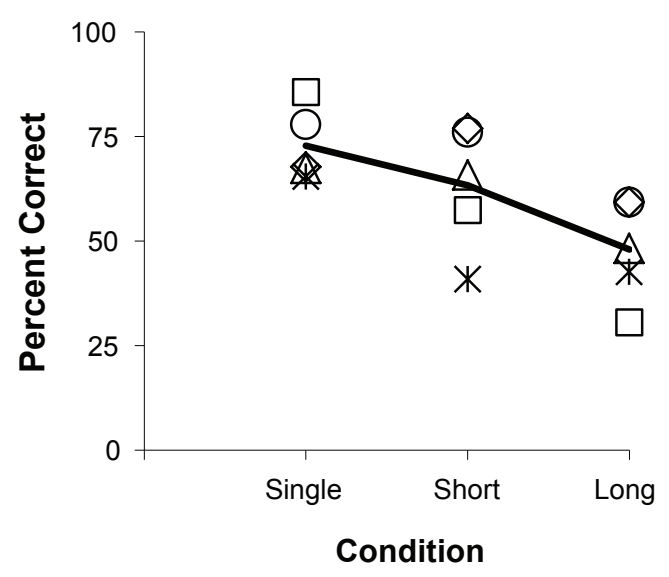

0.5 Letter Spaces

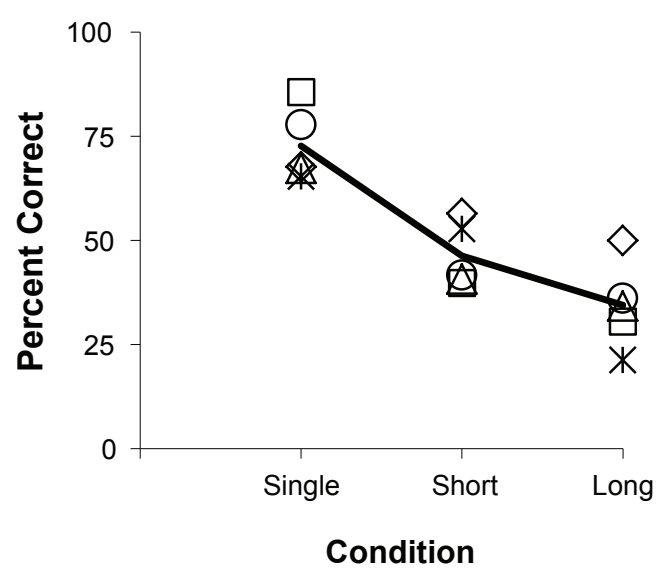


Figure 2

\section{Straight Ahead Gaze}

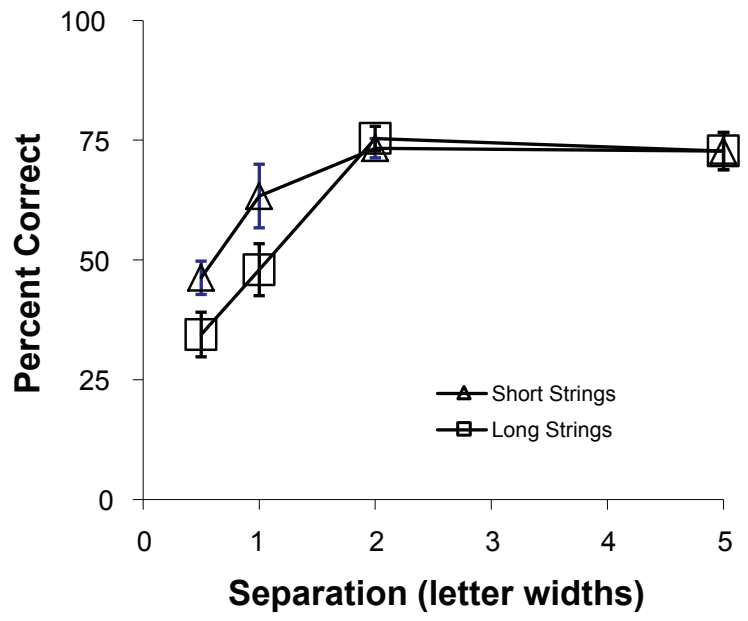

\section{Left Gaze}

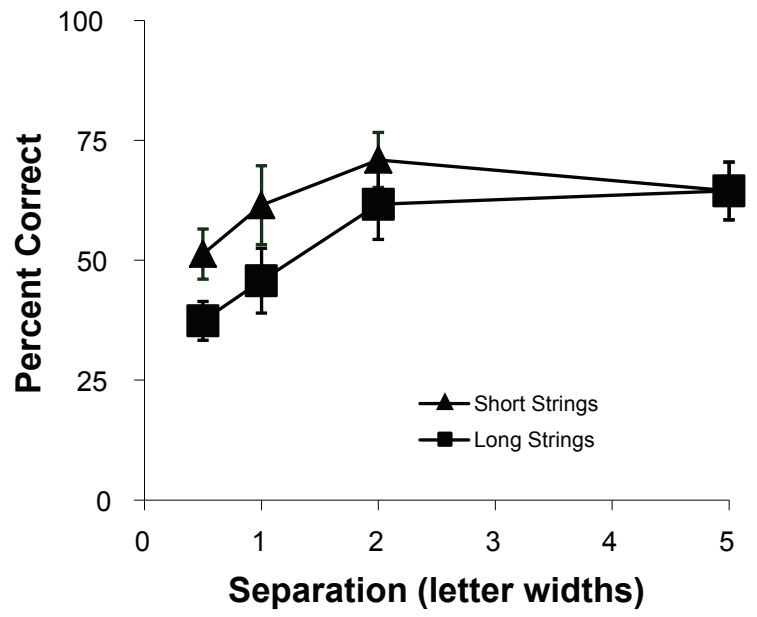


Figyre 3 Trials with Correct Number of Letters

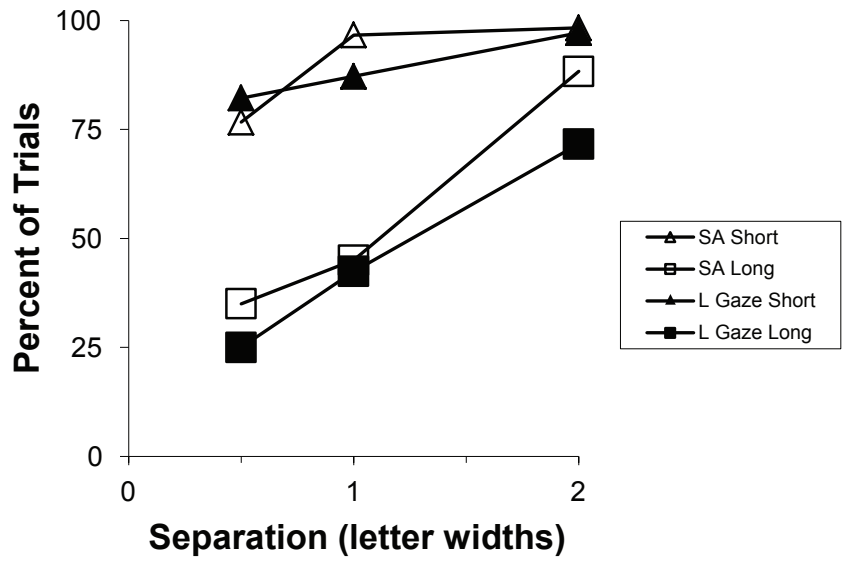


Figure 4

JC: Straight Ahead Gaze

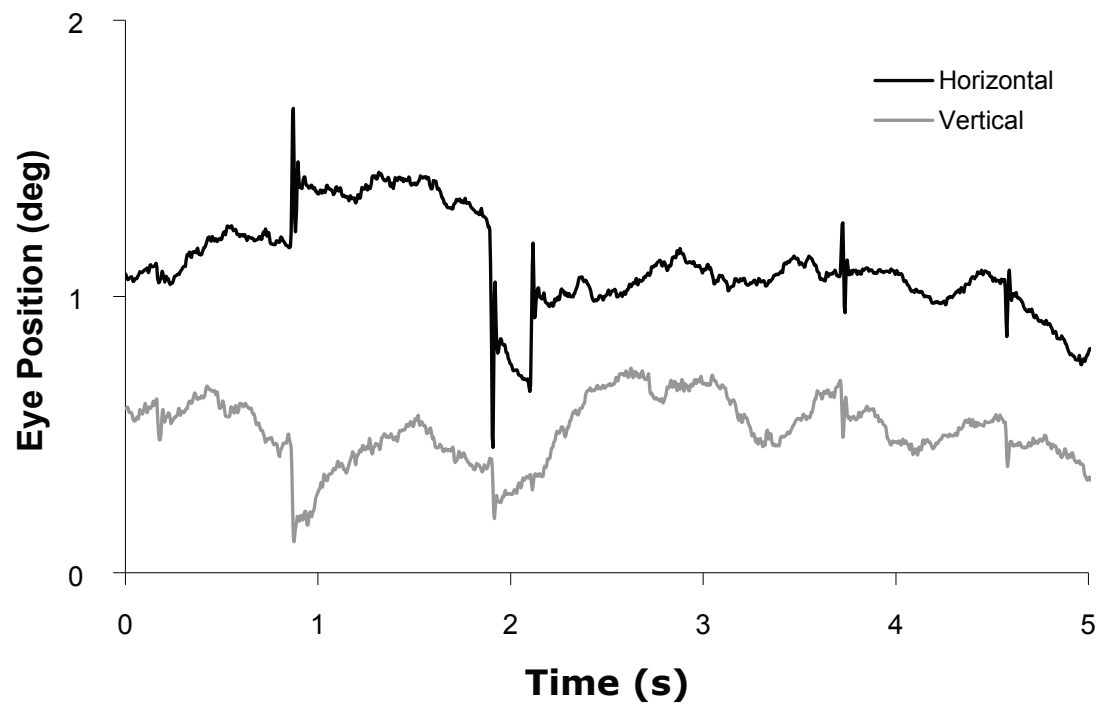

JC: Left Gaze

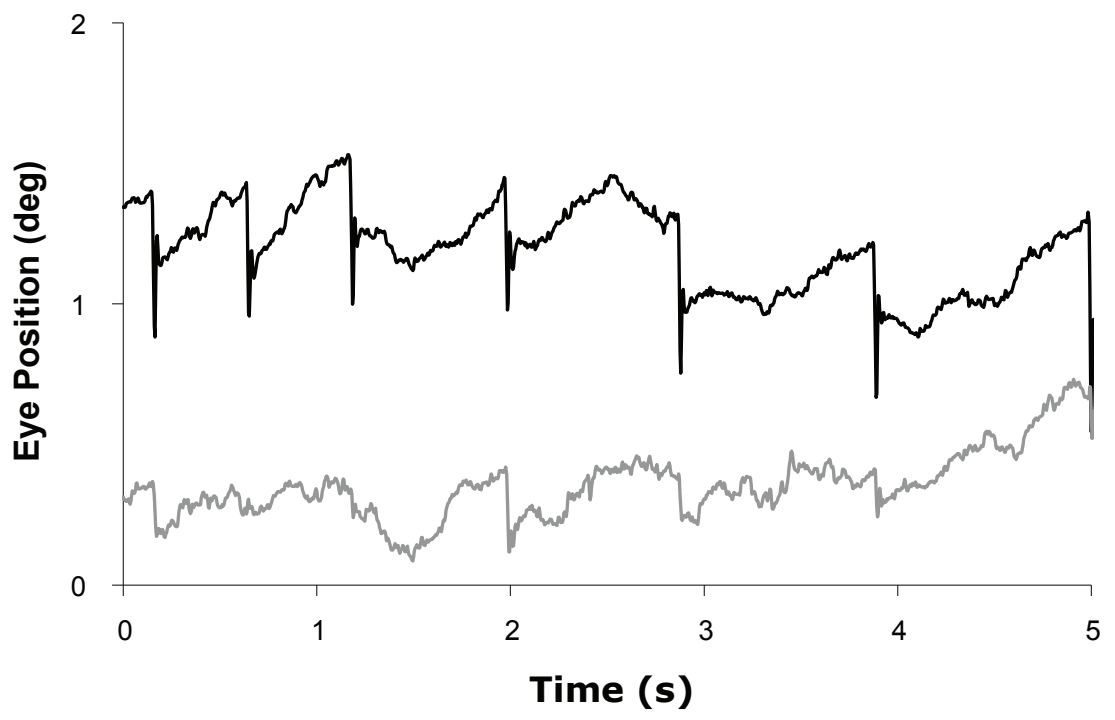


1 Contour interaction is an impairment of fine spatial tasks, such as visual acuity, that occurs in

2 the presence of nearby flanking contours. ${ }^{1,2}$ In addition to contour interaction, inaccurate and

3 imprecise eye movements and divided or misplaced attention have been suggested to degrade

4 visual acuity when measured with letter charts in amblyopic eyes. ${ }^{1,3-6}$ For example, Flom et al.

5 reported that amblyopic eyes exhibit shallower psychometric functions and poorer visual acuity

6 using ' $\mathrm{S}$ charts', composed of 8 Landolt $\mathrm{C}$ targets surrounded on all sides by tumbling Es, than

7 for individual Landolt Cs surrounded by flanking bars. ${ }^{1}$ Eyes with normal vision exhibit little or

8 no difference in the psychometric function for these types of acuity targets. ${ }^{1,7}$ In the aggregate,

9 the deleterious influences of contour interaction, eye movements and attention on object

10 discrimination and recognition are referred to as crowding. ${ }^{5}$

11 Additional evidence for an effect of eye movement and/or misplaced attention on the

12 measurement of foveal visual acuity comes from comparisons between standard clinical and

13 repeat letter acuity charts. ${ }^{8}$ Repeat letter charts were developed to eliminate the effect of

14 inaccurate eye positioning on the assessment of visual resolution. Indeed, measured visual acuity

15 typically is better using repeat letter charts compared to standard clinical charts in populations

16 with abnormal eye movement control, such as observers with amblyopia, ${ }^{9,10}$ nystagmus, ${ }^{11,12}$

17 central field loss, ${ }^{13,14}$ and young children. ${ }^{8,15}$ In contrast, adults with normal vision exhibit only a

18 very slight acuity improvement, on the order of 1 to 1.5 letters, on repeat compared to standard

19 letter charts. ${ }^{9,11,14}$ Instead of an eye-movement effect, this small acuity improvement in adults

20 could be attributable to probability summation that results from multiple concurrent views of the 21 same letter.

22 In normal adult observers, the range of eye movements during foveal fixation is larger than

23 the spacing between adjacent threshold-sized letters on an acuity chart. ${ }^{16-18}$ Further, the saccadic 
24 eye movements between closely spaced targets within an array typically are neither accurate nor

25 precise. ${ }^{19,20}$ These observations suggest that, during the measurement of visual acuity, normal

26 adults may sometimes fixate on a letter other than the one they are attempting to read. It seems a

27 legitimate question to ask why these fixation errors don't interfere with normal observers' acuity,

28 when measured with standard clinical letter charts compared to repeat letter charts or isolated

29 letters. One reason may be that most modern clinical acuity charts include 5 or fewer letters of

30 the same angular size per line. ${ }^{21-23}$ Normal adults can quickly, confidently, and accurately judge

31 numerosity for up to approximately 5 items within a visual display; ${ }^{24,25}$ they may therefore be

32 able to identify the location of a letter they are attempting to read even when the fovea is directed

33 momentarily somewhere else. For displays that contain more than 5 items, observers are slower

34 to determine numerosity and more likely to misperceive the number of items. ${ }^{24,25}$ These

35 observations suggest that inaccurate fixation and/or misplaced attention within a line of acuity

36 letters may be more likely to produce miscalls if the number of letters on the line is increased.

37 The purpose of this study was to infer the role of eye movements and/or attention in normal

38 foveal crowding by comparing performance for short and long horizontal strings of letters with

39 the same letter-to-letter separation, which should therefore produce equivalent amounts of

40 contour interaction. We assessed foveal letter identification both in straight ahead gaze and while

41 the observers fixated in left lateral gaze, where fixation was expected to become more variable.

42 Objective eye-movement recordings indicated that this latter expectation was fulfilled only 43 partially.

44

45 Materials and Methods.

46 Observers. 
Five adult observers_with normal or corrected-to-normal vision participated after granting voluntary written informed consent. Experimental procedures were reviewed and approved by the Ethics Committee of Anglia Ruskin University. Four of the observers were female (3 students and one older adult, age range 21 - 62 years old), all of whom remained naïve as to the experimental hypotheses that were tested in the study. The fifth, non-naïve observer was author JS, who was 51 years old. All testing was performed monocularly, using the left eye. One of the observers (SA) was emmetropic. The other four observers had refractive corrections in the tested

54 left eye of $-1.75 \mathrm{sph}(\mathrm{JC}),-6.50 /-1.50 \times 100(\mathrm{JH}),-3.00 /-0.50 \times 150(\mathrm{JS})$, and +1.75 sph (NB),

55 which were provided during the experiments by ophthalmic trial lenses. The non-viewing right 56 eye was occluded with an opaque patch.

57 Psychophysical Methods.

58 Observers viewed dark Sloan letters presented on a 19-inch Clinton Monoray monochrome 59 monitor from an optical distance of $9.5 \mathrm{~m}$, after reflection from two mirrors. The screen 60 resolution was 1024 by $76 \underline{8}$ pixels and the frame rate was $120 \mathrm{~Hz}$. At this viewing distance, the 61 dimensions of the monitor screen corresponded to 2.33 by $1.75 \mathrm{deg}$. Background luminance was $62110 \mathrm{~cd} / \mathrm{m}^{2}$ and letter contrast, calculated using the Weber formula, was $-99 \%$. A letter size 63 corresponding to $-0.2 \log \mathrm{MAR}$ (3.16 min arc) was used in all of the experimental conditions, as 64 each observer identified approximately $75 \%$ of the letters of this size correctly in preliminary, 65 uncrowded trials (see below). The observers viewed the letters foveally under two conditions of

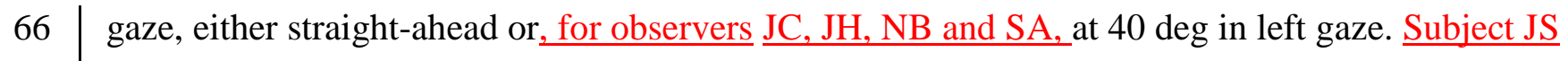
67 could not comfortably sustain fixation at 40 deg left gaze and was tested at 30 deg left gaze instead. For logistical reasons, data collection began in left gaze only after testing in straight-

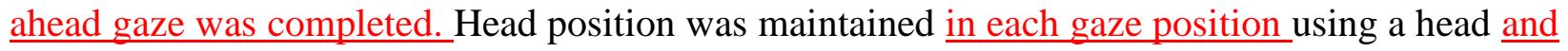


70 chin rest. For the observers who required refractive correction, trial lenses were mounted on a

71 flexible arm, which allowed them to be placed close to the eye and aligned with the visual axis in

72 both straight-ahead and left gaze.

73 Random sequences of Sloan letters, with the stipulation that adjacent letters on the screen

74 were never the same, were presented for an unlimited viewing duration in 3 types of trials. On

75 each short-string trial, 4, 5 or 6 letters were presented at one time, with a horizontal edge-to-edge

76 letter spacing of $0.5,1$ or 2 letter widths (i.e., $1.58,3.16$ or 6.32 min arc). On each long-string

77 trial, a sequence of 10, 11 or 12 letters was presented, also with a horizontal edge-to-edge letter

78 spacing of $0.5,1$ or 2 letter widths. The minimum separation between the edge of the monitor

79 and the edges of the first and last letter in the longest letter strings was 3 letter spaces. For each

80 direction of gaze, observers completed 108 short-string trials (36 each with 4, 5 and 6 letters) and

8136 long-string trials (12 each with 10, 11 and 12 letters). The numbers of short- and long-string

82 trials were chosen so that performance in the two conditions was based on the same total

83 numbers of letters. Short- and long-string trials were presented in blocks of 36 and 12 ,

84 respectively. Within a block of short- or long-string trials the letter-to-letter separation remained

85 constant at $0.5,1$ or 2 letter widths and the strings with different numbers of letters were

86 presented in a random order. Observers were instructed to read all of the letters in each string in

87 sequence from left to right. Although the observers were informed that different numbers of

88 letters would be presented on different trials, they were not told about the length of each letter

89 string either prior to or after a trial. A single trial lasted between approximately 5 and $15 \mathrm{~s}$ and

90 each block of 12 or 36 trials had a total duration of between 5 - 10 minutes. The experimenter

91 used the computer keyboard to record the observers' letter responses in order and subsequently

92 compared the responses to the presented sequences of letters. Because crowding is reduced for 
93 letters at the left and right ends of a line, ${ }^{26,27}$ performance was calculated by scoring only the

94 interior 2, 3 or 4 letters for the trials with 4, 5, and 6 letters, respectively, and only the interior 8,

959 or 10 letters for the trials with 10,11, and 12 letters. Letters had to be read in the correct order

96 to be scored as correct.

97 For comparison with the short- and long-string trials, each observer first completed six

98 blocks (one for each combination of letter separation and gaze direction) of 34 uncrowded trials.

99 On each uncrowded trial, three letters were presented with an edge-to-edge spacing of 5 letter

100 widths (i.e., $15.8 \mathrm{~min}$ arc), a distance well beyond the extent of foveal crowding. ${ }^{1,6,28}$ Because

101 crowding was assumed to be absent in this condition, all 3 letters presented on each trial were

102 scored. As above, the letters had to be read in the correct order to be scored as correct.

103 Eye Movement Recording.

104 To evaluate the influence of gaze direction on fixational eye movements, the horizontal and

105 vertical positions of each observer's left eye were recorded at a sampling rate of $250 \mathrm{~Hz}$ using an

106 EyeLink 1000 Eyetracker with remote camera. Eye movement recordings were obtained in a

107 separate session from the psychophysical measurements. During each trial, the observer fixated

108 for $20 \mathrm{~s}$ at the center of a 3 to 5 letter string, displayed at a distance of $1 \mathrm{~m}$ on a 19-inch Sony

109 Trinitron monitor. Four recordings were made while the observer viewed in straight-ahead gaze

110 and another four were made during foveal fixation at $40 \mathrm{deg}$ (observers JC, JH, NB and SA) or

$11130 \mathrm{deg}$ (observer JS) left gaze. For each direction of gaze, head position was maintained using a

112 head and chin rest.

113 Subsequently, two 5-s intervals without blinks were selected from each fixation file for

114 further analysis: (1) calculation of the standard deviations (SDs) of the horizontal and vertical

115 eye positions during each interval and (2) counts of the number of fixational saccades. The eye 
116 movement results for each observer were summarized by taking the mean horizontal and vertical

117 SDs and the mean number of fixation saccades during the 8 separate fixation intervals (i.e., 4

118 fixation trials $\mathrm{x} 2$ intervals per trial).

119 Data Analysis.

120 To perform statistical analyses, the observers' percent correct letter identification for each

121 experimental condition was converted to a $\mathrm{z}$ score, as between-observer differences in $\mathrm{z}$ scores

122 for the different experimental conditions are more similar than the differences in percent correct.

123 A three-way repeated-measures ANOVA assessed the effects of string length (single letters,

124 short strings and long strings), letter separation (0.5, 1 and 2 letter widths), and gaze direction

125 (straight-ahead and left gaze). A separate two-way repeated-measures ANOVA evaluated 126 whether string length and direction of gaze affected the percentage of trials on which the 127 observers reported the correct number of letters. A Huynh-Feldt correction was applied to 128 statistical tests when a departure from sphericity occurred. Although the statistical analyses were 129 performed using $\mathrm{z}$ scores, for ease of presentation the results were transformed back to percent 130 correct letter identification when plotting Figures $1-3$, below.

\section{Results.}

132 Letter Identification.

133 The average $\mathrm{z}$ scores for letter identification in the uncrowded condition were 0.64 in 134 straight-ahead gaze and 0.43 in left gaze, corresponding to $73.8 \%$ and $66.5 \%$ correct, 135 respectively. These percentages did not differ significantly according to the letter position in the 136 three-letter array (range: $72.0 \%-74.3 \%$ in straight-ahead gaze; $63.4 \%-66.6 \%$ in left gaze), 137 confirming the absence of contour interaction in this condition. When the edge-to-edge 138 separation between adjacent letters was 2 letter spaces, performance in the short- and long-string 
139 conditions did not differ from that in the uncrowded condition (Figure 1 top panels). However,

140 letter identification was poorer when the separation was less than 2 letter spaces, as indicated by

141 a significant effect of separation $\left(\mathrm{F}_{\mathrm{df}=2,8}=16.95, \mathrm{p}=0.0013\right)$. The ANOVA also revealed a

142 significant 2 -way interaction between letter separation and string length $\left(\mathrm{F}_{\mathrm{df}=4,16}=10.53, \mathrm{p}=\right.$

143 0.011). Compared to the uncrowded condition, $\mathrm{z}$ scores were lower for long letter strings when

144 the separation was 1 letter space $\left(\mathrm{F}_{\mathrm{df}=1,16}=13.19, \mathrm{p}=0.019\right)$, and for both the long and short

145 letter strings when the separation was 0.5 letter spaces (for long letter strings, $F_{d f=1,16}=61.81, p=$

146 0.0006; for short letter strings, $\left.F_{d f=1,16}=29.08, p=0.0039\right)$. These effects are visible in the data

147 collected both in straight-ahead and left gaze in the middle and lower panels of Figure 1. Figure

1482 replots the percent correct letter identification in straight ahead and left gaze, averaged across

149 subjects, as a function of the letter separation and shows clearly that the magnitude of crowding

150 is greater for the long compared to short letter strings.

151 Neither the direction of gaze nor the interactions between gaze direction and letter separation

152 or string length exhibited a significant effect on letter identification. However, the ANOVA

153 revealed a significant 3-way interaction between direction of gaze, letter separation, and string-

154 length $\left(\mathrm{F}_{\mathrm{df}=4,16}=3.03, \mathrm{p}=0.049\right)$. Means comparisons indicated that this interaction resulted

155 from significantly better performance in straight-ahead compared to left gaze for long strings

156 when the letter separation was 2 letter widths $\left(\mathrm{F}_{\mathrm{df}=1,16}=13.56, \mathrm{p}=0.0020\right)$.

157 In a second analysis, we compared the percentage of trials (again, converted to $\mathrm{z}$ scores) on

158 which the observers reported the correct numbers of letters in the short- and long-string

159 conditions. As anticipated, a three-way repeated-measures ANOVA found that observers

160 reported the correct number of letters less frequently on long-string than short-string trials $\left(\mathrm{F}_{\mathrm{df}=1,4}\right.$

$161=145.96, \mathrm{p}=0.0003)$. Observers also reported the correct number of letters less often as the 
162 separation between adjacent letters decreased from 2 to 0.5 letter widths $\left(\mathrm{F}_{\mathrm{df}=1,4}=26.41, \mathrm{p}=\right.$

163 0.0014). These trends can be seen in the results for both straight-ahead and left gaze, which are

164 plotted in Figure 3. Of particular interest is the observation that observers reported the correct

165 number of letters on a significantly lower proportion of long-compared to short-string trials even

166 with a separation of two letter widths $\left(\mathrm{F}_{\mathrm{df}=1,8}=12.59, \mathrm{p}=0.0075\right)$. Although the observers

167 generally reported the correct number of letters less frequently in left lateral compared to 168 straight-ahead gaze (Figure 3 ), this difference did not achieve statistical significance $\left(\mathrm{F}_{\mathrm{df}=1,4}=\right.$ $1696.05, \mathrm{p}=0.070)$. More detailed information is presented in Table 1 , which summarizes the 170 percentage of trials on which the observers reported fewer or more than the correct number of 171 letters for each of the experimental conditions. Overall, when the observers did not report the 172 correct number of letters, they erred more often $(\sim 60: 40)$ by reporting too few rather than too 173 many letters, both for the short and long letter strings.

174 When the observers reported too few letters, the majority of the time they reported one letter too few (73\% of errors for long strings; $97 \%$ of errors for short strings). Similarly, when the observers reported too many letters, on the majority of trials they reported one extra letter (88\% of trials for long strings; $98 \%$ of trials for short strings). On the remainder of the trials when an incorrect number letters was reported, the observers either omitted or added 2 (13\% of trials) or 3 letters $(1.6 \%$ of trials), primarily on long-string trials. Examination of the responses on trials when the observers reported the incorrect number of letters indicated that errors of omission or insertion occurred almost always at interior positions in the string. The modal locations of these errors were letter places 6 (omissions) and 8 (insertions) in the long strings (accumulated across strings of 10,11 and 12 letters), and place 4 $\underline{\text { (omissions and insertions) in the short letter strings (accumulated across strings of 4, 5, and } 6}$ 
letters). When the observers reported one or more extra letters within a string, they typically reported a pair of confusion letters for one letter within the string, such as both ' $N$ ' and ' $R$ ' for the occurrence of an 'R' or both ' $\mathrm{D}$ ' and ' $\mathrm{C}$ ' for the occurrence of an 'O.'

Observers would be expected to make more errors in reporting the correct number of letters for long compared to short letter strings, simply because the long strings include more letters to report. However, the following analysis reveals that merely increasing the average number of letters in the string does not account for our results. Assume the probability of incorrectly reporting each letter, either by omitting the letter or reporting an additional letter, is the same regardless of the string length. Further, assume that this probability, which we will designate as $\mathrm{p}_{\mathrm{e}}$, depends on the separation between the adjacent letters in the string. For each letter separation, we can estimate the value of $p_{e}$ from the percentage of reporting errors made when reading short letter strings, $\mathrm{p}_{5}$. Specifically, if each short letter string is assumed to comprise 5 letters, then

$$
\left(1-p_{e}\right)^{5}=1-p_{5}
$$

and the predicted percentage of errors when reading strings of 11 letters, $\mathrm{p}_{11}$, is

$$
\mathrm{p}_{11}=1-\left(1-\mathrm{p}_{\mathrm{e}}\right)^{11}
$$

For example, when the letter separation is 2 letters in straight-ahead gaze, summing the percentages of reporting too few and too many letters in the short-string condition yields $\mathrm{p}_{5}=$ 0.023 (Table 1) and $\left(1-p_{5}\right)=0.977$. Based on this result, the predicted probability of reporting an incorrect number of letters in the analogous long-string condition, $\mathrm{p}_{11}$, is 0.05 . The observed probability of reporting the incorrect number of letters in this long-string condition was 0.15 , which is significantly higher $(\mathrm{z}=3.55, \mathrm{p}=0.0004)$ than the predicted value. The observed probabilities of reporting the incorrect numbers of letters in the long-string condition also is 
207 significantly greater than the predicted probabilities $(\mathrm{p}<0.0001)$ for the other 5 conditions of

208 letter separation and gaze direction.

209 Fixational Eye Movements.

210 During fixation in straight-ahead gaze, the average standard deviations (SD) of both the

211 horizontal and the vertical eye position measurements were approximately $0.16 \mathrm{deg}$ (i.e., $9.5 \mathrm{~min}$

212 arc; Table 2). Although four of the five observers (all except JH) exhibited a small endpoint

213 nystagmus when fixating in left gaze (Figure 4), only two of them exhibited a noticeable increase

214 in the SD of horizontal eye position: for observer JS from 0.13 to $0.19( \pm 0.02 \mathrm{SE})$ deg and for

215 observer NB from 0.19 to $0.27( \pm 0.02 \mathrm{SE})$ deg. The SDs of vertical eye position were either

216 unchanged in left compared to straight-ahead gaze or decreased (from 0.24 to 0.11 deg for

217 observer JC). However, consistent with the observed endpoint nystagmus in left gaze, the

218 average number of saccades during fixation in left gaze was significantly greater than the number 219 of saccades during fixation in straight-ahead gaze (mean difference $=2.58$ saccades $/ 5 \mathrm{~s}, \mathrm{t}_{\mathrm{df}=4}=$ $2203.10, \mathrm{p}=0.036)$.

221 We compared the change in correct letter identification between straight-ahead and left gaze

222 in observers JS and NB, who showed an increase in the SD of horizontal eye position, to the

223 change in performance of the other three observers. We focused on the change in $\mathrm{z}$ scores for

224 long letter strings separated by two letter widths because, as reported above, this condition

225 produced a significant gaze-dependent effect on crowding. The average change in z score from

226 straight ahead to left gaze was $0.75 \pm 0.08$ for JS and NB, compared to $0.10 \pm 0.19$ for the other

227 three observers.

228 Discussion. 
In normal observers, contour interaction does not occur in the fovea for targets separated by

230 more than 3-6 min arc, which corresponds approximately to the width of a high-contrast

231 threshold acuity letter. ${ }^{1,2,7,29-31}$ On this basis, we conclude that only the immediately adjacent

232 letters within each string contributed to contour interaction and that the magnitude of contour

233 interaction was identical for the interior letters in the short- and long-string stimuli in our

234 experiment. The greater magnitude of crowding that we observed for strings of 10, 11 and 12

235 letters than for strings of 4, 5 and 6 letters for a separation of 0.5 and 1 letter space (Figure 2)

236 therefore can not be attributed to an increase in contour interaction.

237 Inaccurate and imprecise eye movements are a potential contributor to foveal crowding. ${ }^{1,5,8,32}$

238 The imprecision of normal fixation is large enough to encompass more than a single letter, even

239 in the letter strings with a separation of two letter spaces between the adjacent letters ${ }^{16-18}$ (see

240 also Figure 4). Moreover, it is highly unlikely that our observers accurately imaged the

241 successive letters in the strings sequentially on the fovea. Kowler and Steinman ${ }^{20}$ measured the

242 saccades made by two experienced observers when counting 10 to 19 identical vertical lines

243 separated horizontally by gaps of $7.4-14.2 \mathrm{~min}$ arc. For lines separated by 7.4 to $8.2 \mathrm{~min}$ arc,

244 one observer made saccades that ranged in amplitude from approximately 7 to 45 min arc; the

245 saccades of the other observer ranged from less than 5 to approximately 20 min arc. Both

246 observers frequently lost their place while counting the vertical lines and, for the range of line

247 separations examined, reported the correct number of lines on half or fewer of the trials. Our

248 finding that observers often reported the incorrect number of letters in the long-string condition

249 (Fig. 3) is consistent with this observation. As suggested above in the Introduction, observers

250 may be less likely to lose their place in short letter strings because they can judge the number of 
251 letters, and presumably their relative positions in the string, essentially instantaneously and 252 effortlessly. ${ }^{24,25}$

253 Liu and Arditi observed that normal observers frequently reported only 4 of the letters in 254 strings of 5 upper case letters when the edge-to-edge letter-to-letter spacing was made very 255 small. $^{33}$ The frequency of these under-reporting errors dropped rapidly from approximately $80 \%$ 256 to $20 \%$ as the separation between adjacent letters increased from 0.04 to 0.24 of the threshold 257 letter size. Only rarely did the observers make the opposite error of reporting 5 letters in strings 258 that were 4 letters in length. Based on the strong dependence of under-reporting errors on the 259 letter separation, the authors attributed these errors to overlap between the retinal images of 260 adjacent letters. The observers in our study often erred in reporting the correct number of letters 261 for edge-to-edge separations of 0.5 and 1 letter width, well beyond the range of separations used 262 by Liu and Arditi. ${ }^{33}$ In addition, both under- and over-reporting of the number of letters were 263 common among our observers (Table 1). For these reasons, we conclude that our results can not 264 be attributed to overlap between the images of adjacent letters.

265 It is possible that the observers used the angular extent subtended by each string as a cue to 266 the number of letters, as the ratios of the extent for the different short and long strings presented 267 within a single block of trials generally exceed the Weber fraction for length discrimination (2 268 5\%, e.g., Helmholtz, 1925; Ono, $1967^{34, \underline{35}}$ ). For the three letter separations tested, the ratios of 269 extent for the 4, 5, and 6 letter strings are greater than those between 10, 11, and 12 letter strings, 270 which potentially could account for the higher percentage of errors in reporting the number of 271 letters on long- compared to short-string trials. However, the ratios of angular extent for the 272 short and long letter strings do not change appreciably with the letter separation; i.e., the angular 273 extent of 6 and 4 letter strings are in the ratio of 1.60 for a letter separation of two spaces and 
2741.55 for a letter separation of 0.5 spaces. Similarly, the angular extent of 12 and 10 letter strings

275 are in the ratio of 1.21 for letter separations of both two and 0.5 spaces. Nevertheless, the

276 number-of-response errors increase as the letter separation decreases from two to 0.5 spaces,

277 both for the short and long letter strings (Figure 3). Further, in addition to the angular extent of

278 the letter strings, information about the string length on each trial was available also from the

279 angular separation between the first and last letter of the string and the edges of the monitor

280 screen. The ratios of these separations for the different string lengths are uniformly higher for

281 long than for short letter strings, and are greatest for long strings when the letter separation is

282 large. When the separation between the adjacent letters in the long strings was two spaces, the

283 ratios of the distances to the monitor edge for strings of 10, 11, and 12 letters are essentially

284 identical to the ratios of the angular extents for strings of 4,5 , and 6 letters separated by one

285 space. Nevertheless, observers made approximately 4.5 times more number-of-response errors

286 on long-string trials with a separation of two letter spaces than on short-string trials with a

287 separation of one letter space. The observations in this paragraph suggest that the observers did

288 not attempt to deduce the number of letters presented on each trial and that, for a given

289 separation between letters, the more frequent errors for long compared to short strings in the

290 number of reported letters is attributable primarily to the number of letters the in the string.

291 We expected that observers would make more errors when they viewed the letter strings at

29230 or 40 deg in left gaze compared to straight ahead because of an increase in the variability of

293 fixation in association with the development of endpoint nystagmus. ${ }^{36}-3 \underline{8}$ Although we observed a

294 trend for a greater number of miscalls and more frequent errors in reporting the number of letters

295 in a string, these trends did not reach statistical significance. The likely reason for the observers'

296 overall similar psychophysical performance in left and straight-ahead gaze is that the variability 
297 of horizontal fixation did not change significantly in left gaze for three of the five observers. The

298 two observers whose horizontal fixation was less stable in left gaze also identified widely spaced

299 letters in the long-string condition less accurately in left compared to straight-ahead gaze. It is

300 possible that fixation would have been less steady and psychophysical performance would have

301 been worse if the observers had been required to view the letters with gaze diverted further to 302 one side. ${ }^{3 \underline{8}}$

303 A displacement of attention from the acuity target towards the flanking stimuli also has been

304 suggested to contribute to crowding. ${ }^{1,5}$ Leat et al. sought to assess the influence of attention on

305 crowding by varying the similarity of the flanking targets to one another and to the acuity target

306 which, in their study, was a Landolt $\mathrm{C}$ or tumbling E. ${ }^{6}$ The results indicated that the manipulation

307 of flanker characteristics generates no consistent change in the magnitude of foveal crowding.

308 However, it may be difficult to tease apart the potentially separate influences of eye movements

309 and attention on foveal crowding, as there is evidence to suggest that the locus of attention

310 remains closely tied to the direction of gaze during large eye movements. ${ }^{32-41}$ A similar intimate

311 relationship has been suggested to exist also between the direction of gaze and attention during

312 small shifts in the position of fixation by some, ${ }^{42-44}$ but not all authors. ${ }^{45}$

313 We conclude that both the greater magnitude of foveal crowding and more frequent errors in

314 reporting the correct number of letters in long compared to short letter strings are attributable to

315 the imprecision and inaccuracy of small normal eye movements. As surmised previously by

316 others, the eye-movement contribution to crowding may be substantially greater in individuals

317 whose eye-movement control is poor, such as in patients with nystagmus, ${ }^{11,12}$ amblyopia, ${ }^{1,5,9,10}$

318 and young children. , $^{8,15,32}$

319 Acknowledgments. 
320 This research was supported by a grant from the Evelyn Trust and a Leverhulme Visiting

321 Professorship to Anglia Ruskin University. Portions of the data were presented at the annual

322 meeting of the American Academy of Optometry in Seattle, WA in October, 2013.

323 


\section{References.}

325 1. Flom MC, Weymouth FW, Kahneman D. Visual resolution and contour interaction. Journal of the 326 Optical Society of America, 1963; 53:1026-32.

327 2. Takahashi ES. Effects of flanking contours on visual resolution at foveal and near-foveal loci. PhD

328 Thesis: School of Optometry, Berkeley: University of California;1968.

329 3. Adler FH. Physiology of the Eye: Clincal Application, $3^{\text {rd }}$ Edition. St. Louis, Mosby, 1959:697-8.

330 4. Flom MC. New concepts in visual acuity. Optometric Weekly 1966; 57(28): 63-8.

331 5. Flom MC. Contour interaction and the crowding effect. Problems in Optometry 1991; 3: 237-57.

332 6. Leat SJ, Li W, Epp K. Crowding in central and eccentric vision: the effects of contour interaction and 333 attention. Investigative Ophthalmology and Visual Science 1999; 40: 504-12.

334 7. Danilova MV, Bondarko VM Foveal contour interactions and crowding effects at the resolution limit of the visual system. Journal of Vision 2006; 7(2); 25:1-18.

8. Kothe AC, Regan D. Crowding depends on contrast. Optometry and Vision Science 1990; 67: 283-6.

9. Regan D, Giaschi DE, Kraft SP, Kothe AC. Method for identifying amblyopes whose reduced line acuity is caused by defective selection and/or control of gaze. Ophthalmic \& Physiological Optics, 1992; 12: 425-32.

10. Simmers AJ, Gray LS, McGraw PV, Winn B. Functional vision loss in amblyopia and the effect of occlusion therapy. Investigative Ophthalmology \& Visual Science 1999; 40: 2859-71.

342 11. Simmers AJ, Gray LS, Winn B. The effect of abnormal fixational eye movements upon visual acuity in congenital nystagmus. Current Eye Research 1999; 18: 194-202.

344 12. Park H, Robins D, Guyton DL. A new way to measure potential visual acuity in nystagmus patients:

345 full-field E test. Investigative Ophthalmology \& Visual Science 2010; 51, E-Abstract \#1996.

346 13. Harris MJ, Robins D, Dieter Jr JM, Fine SL, Guyton DL. Eccentric visual acuity in patients with 347 macular disease. Ophthalmology 1985; 92: 1550-3.

348 14. Pardhan S. Crowding in visually inpaired patients: contour interaction and/or gaze selection defects? $349 \quad$ Neuro-ophthalmology 1997; 18: 59-65.

350 15. Simmers AJ, Gray LS, Winn B. Visual function thresholds in children. Current Eye Research 2000; $351 \quad 21: 616-26$.

352 16. Cherici C, Kuang X, Poletti M, Rucci M. Precision of sustained fixation in trained and untrained 353 observers. Journal of Vision 2012; 12(6);31: 1-16. 
354 17. Ferman L, Collewijn H, Jansen TC, Van den Berg AV. Human gaze stability in the horizontal,

355 vertical and torsional direction during voluntary head movements, evaluated with a three-dimensional

356 scleral induction coil technique. Vision Resesarch 1987; 27: 811-28.

357 18. Skavenski AA, Hansen RH, Steinman RM, Winterson B. Quality of retinal image stabilization during 358 small natural and artificial body rotations in man. Vision Research 1979; 19: 675-83.

359 19. He P, Kowler E. The role of saccades in the perception of texture patterns. Vision Research 1992; 32: $360 \quad 2151-63$.

361 20. Kowler E, Steinman RM The role of small saccades in counting. Vision Research 1977; 17: 141-6.

362 21. Bailey IL, Lovie JE. New design principals for visual acuity letter charts. American Journal of 363 Optometry \& Physiological Optics 1976; 53: 740-5.

364 22. Ferris $3^{\text {rd }}$ FL, Kassoff A, Bresnick GH, Bailey I. New acuity charts for clinical research. American 365 Journal of Ophthalmology, 1982; 94: 91-6.

366 23. Hartmann EE, Dobson V, Hainline L, Marsh-Tootle W, Quinn GE, Ruttum MS, Schmidt PP, Simons

367 K. Preschool vision screening: summary of a task force report. Pediatrics, 2000; 106: 1105-12.

368 24. Kaufman EL, Lord MW, Reese TW, Volkmann J. The discrimination of visual number. American 369 Journal of Psychology 1949; 62: 498-525.

370 25. Li R, MacKeben M, Chat SW, Kumar M, Ngo C, Levi DM. Aging and visual counting. PLoS One $371 \quad 2010 ; 5 ; \mathrm{e} 13434: 1-8$.

372 26. Mathew JA, Shah SS, Simon JW. Varying difficulty of Snellen letters and common errors in 373 amblyopic and fellow eyes. Archives of Ophthalmolgy 2011; 129: 184-7.

374 27. Stuart JA, Burian HM. A study of separation difficulty. Its relationship to visual acuity in normal and 375 amblyopic eyes. American Journal of Ophthalmology 1962; 53: 471-7.

376 28. Hess RF, Dakin SC, Kapoor N, Tewfik M. Contour interaction in fovea and periphery. Journal of the 377 Optical Society of America A 2000; 17: 1516-24.

378 29. Bedell HE, Siderov J, Waugh SJ, Zemanova R, Pluhacek F, Musilova L. Contour interaction for 379 foveal acuity targets at different luminances. Vision Research 2013; 89: 90-5.

380 30. Siderov J, Waugh SJ, Bedell HE. Foveal contour interaction for low contrast acuity targets. Vision $381 \quad$ Research 2013; 77: 10-3.

382 31. Lev M, Yehezkel O, Polat U. Uncovering foveal crowding? Scientific Reports 2014; 4: 1-6.

383 32. Norgett Y, Siderov J. Crowding in children's visual acuity tests - effect of test design and age. Optometry \& Vision Science 2011; 88: 920-27. 
33. Liu L, Arditi A. Apparent string shortening comcomitant with letter crowding. Vision Research 2000; 40: 1059-67.

34. von Helmholtz, H. Treatise on Physiological Optics. Volume III. The Perceptions of Vision. Southall, JPC, trans. New York: Optical Society of America; 1925: 169-170, 232-233.

35. Ono, H. Difference thresholds for stimulus length under simultaneous and nonsimultaneous viewing conditions. Perception \& Psychophysics 1967; 2: 201-207.

36. Abel LA, Parker L, Daroff RB, Dell'Osso LF. End-point nystagmus. Investigative Ophthalmology \& Visual Science 1978; 17, 539-44.

37. Eizenman M, Cheng P, Sharpe JA, Frecker RC. End-point nystagmus and ocular drift: an experimental and theoretical study. Vision Research 1990; 30: 863-77.

38. Shallo-Hoffmann J, Schwarze H, Simonsz HJ, Mühlendyck H. A rexamination of end-point and rebound nysatgmus. Investigative Ophthalmology \& Visual Science 1990; 31: 388-92.

39. Rizzolatti G, Riggio L, Dascola I, Umiltá, C. Reorienting attention across the horizontal and vertical meridians: evidence in favor of a premotor theory of attention. Neuropsychologia 1987; 25: 31-40.

40. Kowler E, Anderson E, Dosher B, Blaser E. The role of attention in the programming of saccades. Vision Research 1995; 35: 1897-1916.

41. Deubel H, Schneider WX. Saccade target selection and object recognition: evidence for a common attentional mechanism. Vision Research 1996; 36: 1827-37.

42. Engbert R, Kliegl R. Microsaccades uncover the orientation of covert attention. Vision Research 2003; 43: 1035-45.

43. Hafed ZM, Clark JJ. Microsaccades as an overt measure of covert attention. Vision Research 2002; 42: 2533-45.

44. Hafed ZM, Lovejoy LP, Krauzlis RJ. Modulation of microsaccades in monkey during a covert visual attention task. Journal of Neuroscience 2011; 31: 15219-30.

4․ Horowitz TS, Fine EM, Fencsik DE, Yurgenson S, Wolfe JM. Fixational eye movements are not an index of attention. Psychological Science 2007; 18: 356-63. 
412 Table 1. Percentages of trials on which the five observers reported too few vs. too many letters

413 for different combinations of string length, letter spacing, and gaze direction.

414

Letter Type of Straight-ahead Gaze Left Gaze

Separation Error Short strings Long strings Short strings Long strings

\begin{tabular}{|c|c|c|c|c|c|}
\hline \multirow[t]{2}{*}{0.5} & too few & $18.9 \%$ & $40.7 \%$ & $10.6 \%$ & $45.0 \%$ \\
\hline & too many & $4.4 \%$ & $20.0 \%$ & $7.2 \%$ & $30.0 \%$ \\
\hline \multirow[t]{2}{*}{1} & too few & $2.2 \%$ & $35.0 \%$ & $1.7 \%$ & $36.7 \%$ \\
\hline & too many & $1.1 \%$ & $20.0 \%$ & $4.4 \%$ & $16.7 \%$ \\
\hline \multirow[t]{2}{*}{2} & too few & $0.6 \%$ & $1.7 \%$ & $0.6 \%$ & $15.0 \%$ \\
\hline & too many & $1.7 \%$ & $13.3 \%$ & $2.2 \%$ & $13.3 \%$ \\
\hline
\end{tabular}

415

416

417

418

419

420

Table 2. Mean standard deviations (SDs) of horizontal and vertical eye position during fixation for five observers in straight-ahead and left gaze.

\begin{tabular}{|c|c|c|c|c|}
\hline \multirow[b]{2}{*}{ Observer } & \multicolumn{2}{|c|}{ Straight-ahead Gaze } & \multicolumn{2}{|l|}{ Left Gaze } \\
\hline & Horizontal SD & Vertical SD & Horizontal SD & Vertical SD \\
\hline $\mathrm{JC}$ & $0.16 \pm 0.05$ & $0.24 \pm 0.09$ & $0.14 \pm 0.04$ & $0.11 \pm 0.02$ \\
\hline $\mathrm{JH}$ & $0.17 \pm 0.05$ & $0.17 \pm 0.03$ & $0.17 \pm 0.04$ & $0.15 \pm 0.01$ \\
\hline JS & $0.13 \pm 0.04$ & $0.11 \pm 0.04$ & $0.19 \pm 0.03$ & $0.14 \pm 0.06$ \\
\hline NB & $0.19 \pm 0.03$ & $0.10 \pm 0.04$ & $0.27 \pm 0.04$ & $0.13 \pm 0.04$ \\
\hline SA & $0.17 \pm 0.05$ & $0.18 \pm 0.11$ & $0.13 \pm 0.03$ & $0.17 \pm 0.03$ \\
\hline
\end{tabular}




\section{Figure Legends.}

424

Figure 1. Percent correct letter identification is plotted for 5 observers for 3 presentation conditions of foveal viewing: uncrowded ('single') letters, short-letter strings, and long-letter strings. The top, middle, and lower pairs of panels show data for edge-to-edge letter separations of 2, 1 and 0.5 letter spaces. The right and left columns present results for letter strings viewed in straight ahead and left gaze, respectively. Within each column, the same values are plotted for each observer in the three panels for the uncrowded condition. The black lines in each panel connect the mean values of percent correct for the 3 different presentation conditions.

Figure 2. Mean percent correct letter identification is plotted as a function of the edge-to-edge letter spacing for short- and long-letter strings. The values plotted for a separation of 5 letter widths are from the uncrowded condition. The top and bottom panels present results for letters viewed in straight-ahead and left gaze.

Figure 3. The percentages of trials on which the observers reported the correct numbers of letters in the short and long letter strings are plotted as a function of the edge-to-edge letter separation within the strings. Results are shown separately for straight-ahead (unfilled symbols) and left gaze (filled symbols).

Figure 4. Five-second recordings of horizontal (black, higher traces) and vertical (gray, lower traces) eye position during fixation in straight-ahead (top) and left gaze (bottom) are presented for observer JC. The placement of each trace with respect to the vertical axis is arbitrary. Upward deflections represent rightward and upward eye movement. A leftward endpoint nystagmus with an amplitude of approximately one third of a degree is visible in the lower panel. 